\title{
Adsorption and Hybridization of Oligonucleotides on Mercaptoacetic Acid Capped CdSe/ZnS Quantum Dots and Quantum Dot-Oligonucleotide Conjugates
}

\author{
W. Russ Algar, Ulrich J. Krull* \\ Chemical Sensors Group, Department of Chemical and Physical Sciences, University of \\ Toronto at Mississauga, 3359 Mississauga Road North, Mississauga, Ontario, L5L 1C6, \\ Canada
}

*Corresponding author. Tel.: +1 905828 5437; fax: +1 905828 5425. E-mail address: ukrull@utm.utoronto.ca (U.J. Krull)

\section{SUPPORTING INFORMATION}

\section{Quantum Dot Capping and Quantum Dot-DNA Conjugate Preparation}

QDs in toluene were made water soluble by ligand exchange with mercaptoacetic acid (MAA). In a typical procedure, QDs in toluene were diluted in chloroform with $>5 \times 10^{4}$ fold excess of MAA and sufficient $\mathrm{N}, \mathrm{N}$-diisopropylethylamine to render the solution basic. The mixture was sonicated for 2-3 minutes and refluxed under argon for 8-12 hours. During this period the QDs precipitated. The precipitate and supernatant were centrifuged to produce a compact pellet, the supernatant discarded, and the precipitate was washed three times with chloroform. Each wash consisted of mixing, 
centrifuging, and discarding the supernatant. After drying in air to remove residual chloroform, the precipitate was dissolved in TB buffer. This was followed by reprecipitation via the addition of $95 \%$ ethanol (ca. 3:1 EtOH:TB) and centrifugation. This step was repeated once more prior to dissolving the water soluble quantum dots (MAAQDs) in the desired amount of TB buffer. The concentration of MAA-QDs was determined by absorption spectroscopy using the first absorption peak at $515 \mathrm{~nm}$.

DNA conjugates were prepared by mixing MAA-QDs with $n$ equivalents of amine modified oligonucleotides in TB buffer containing EDC. The mixture was allowed to stand 6-8 $\mathrm{h}$ at room temperature. Following the reaction, excess EDC was removed by precipitation of the QD-DNA conjugate with ethanol, centrifugation, and discarding the supernatant. The conjugates were then redissolved in TB buffer and precipitated with ethanol twice more before finally dissolving in the desired amount of buffer. Typical conjugate recoveries were $70-80 \%$ and final concentrations were determined by UVvisible absorption spectroscopy. QD-DNA conjugates were centrifuged for $15 \mathrm{~min}$ at 10 $000 \mathrm{rpm}$.

\section{Relative Quantum Yield and Lifetime Measurements}

Relative quantum yields were measured for QDs by comparing the QD luminescence-to-absorbance ratio between samples and the corresponding control, where luminescence was excited at $385 \mathrm{~nm}$ and absorbance was measured at $385 \mathrm{~nm}$. Relative quantum yields for $\mathrm{Cy} 3$ labeled oligonucleotides as QD-conjugates were measured by comparing the $\mathrm{Cy} 3$ fluorescence-to-absorbance ratio to control samples, using $520 \mathrm{~nm}$ for 
fluorescence excitation and absorbance measurements. Ultraviolet-visible absorption was measured using a HP 8452A Diode-Array Spectrometer (Hewlett Packard Corporation, Palo Alto, CA, USA). Luminescence measurements were made using a QuantaMaster PTI Spectrofluorimeter and Felix Software (Photon Technology International, Lawrenceville, NJ, USA).

The relative fluorescence lifetimes for samples were determined by comparing the lifetimes measured for both the $\mathrm{QD}$ and $\mathrm{Cy} 3$ to the lifetimes measured for the corresponding control samples. Photoluminescence lifetime measurements were made using a time correlated single photon counter (constructed in-house), ${ }^{1}$ driven by a $520 \mathrm{~nm}$ femtosecond laser (pulse duration: $200 \mathrm{fs}$, repetition rate: $15 \mathrm{MHz}$, bandwidth: 3nm, mean power: $1 \mathrm{~mW}$ at $520 \mathrm{~nm}$ ). Luminescence decay curves were obtained using SPCM software (Version 8.50) and SPC-630 hardware (Becker \& Hickl GmbH, Berlin, Germany), and analyzed using SPCImage (Version 2.8.3.2921, Becker \& Hickl GmbH). Fluorescence decay curves were fit with a monoexponential lifetime by minimizing the $\chi^{2}$ value. The system response used in the fitting routine was measured experimentally. The initial $5 \mathrm{~ns}$ of the decay curve were not used in the fitting routine to isolate the long lifetime component. A monoexponential decay fit the data and simplified the analysis of FRET efficiency. The QD emission was isolated by using a combination of a $530 \mathrm{~nm}$ long-pass coloured glass filter (Melles Griot, Rochester, NY, USA) and $550 \mathrm{~nm}$ shortpass interference filter (ThorLabs, Newton, NJ, USA). Using a spectrofluorimeter, this was confirmed to block the majority of the Cy3 emission excited directly at $520 \mathrm{~nm}$. Cy3 fluorescence was isolated using a $550 \mathrm{~nm}$ long-pass interference filter (ThorLabs). 


\section{On the Role of Ionic Strength in Adsorption}

The influence of ionic strength on adsorption is observed as a lower adsorption in $15 \mathrm{mM}$ TB buffer compared with $90 \mathrm{mM}$ TB buffer. Furthermore, despite similar ionic strengths, the adsorption in acetate buffer is much greater than adsorption in TB buffer. Similarly, adsorption is weak in borate buffer, despite having an ionic strength five-fold larger than TB or acetate buffer. These two trends indicate the secondary importance of ionic strength with respect to $\mathrm{pH}$.

\section{On the Adsorption of Poly-T (or lack thereof)}

While a homopolymer of adenine (poly-A) behaved similarly to the mixed base sequence (data not shown), a homopolymer of thymine (poly-T) behaved differently. The poly-T sequence showed no tendency to adsorb, except at high $\mathrm{pH}$ where other sequences tended to adsorb the least. However, like the mixed base sequence, the adsorption of poly- $T$ was hindered by the presence of covalently attached oligonucleotides. While this apparent sequence dependence supports the idea that the interactions driving adsorption are related to the nucleobases, the tendency for the poly-T sequence to adsorb preferentially at high $\mathrm{pH}$ is not expected within a hydrogen bonding model. It is unclear that the methyl group of thymine would disrupt hydrogen bonding interactions at any $\mathrm{pH}$. Furthermore, it may also be supposed that partial deprotonation of the thymine would occur in borate buffer at $\mathrm{pH}=9.5$ since this value is close to its acid dissociation constant $\left(\mathrm{pK}_{\mathrm{a} 2}=9.8\right){ }^{2}$ If the failure to adsorb at lower $\mathrm{pH}$ values is due 
to the greater hydrophobicity of thymine, then it is plausible that deprotonation would have an effect on the adsorptive behaviour of poly-T. In this respect the results suggest that, in addition to hydrogen bonding and electrostatic considerations, there may be another underlying force driving adsorption, although its nature is not yet clear. It may also be expected that poly-A/poly-T hybrid would show some adsorption due to the presence of the poly-A sequence. However, it is known that the minor groove of polyA/poly-T hybrids is narrowed, ${ }^{3,4}$ which may hinder adsorptive interactions with the polyA sequence.

\section{Kinetic Traces for Adsorption and Hybridization}

Examples of kinetic curves for adsorption and hybridization are shown in Figure S1. The curves show the change in FRET sensitized Cy3 fluorescence with time and can be fit with the exponential function (equation 1 in article text), where a kinetic parameter can be extracted and is of interest. 


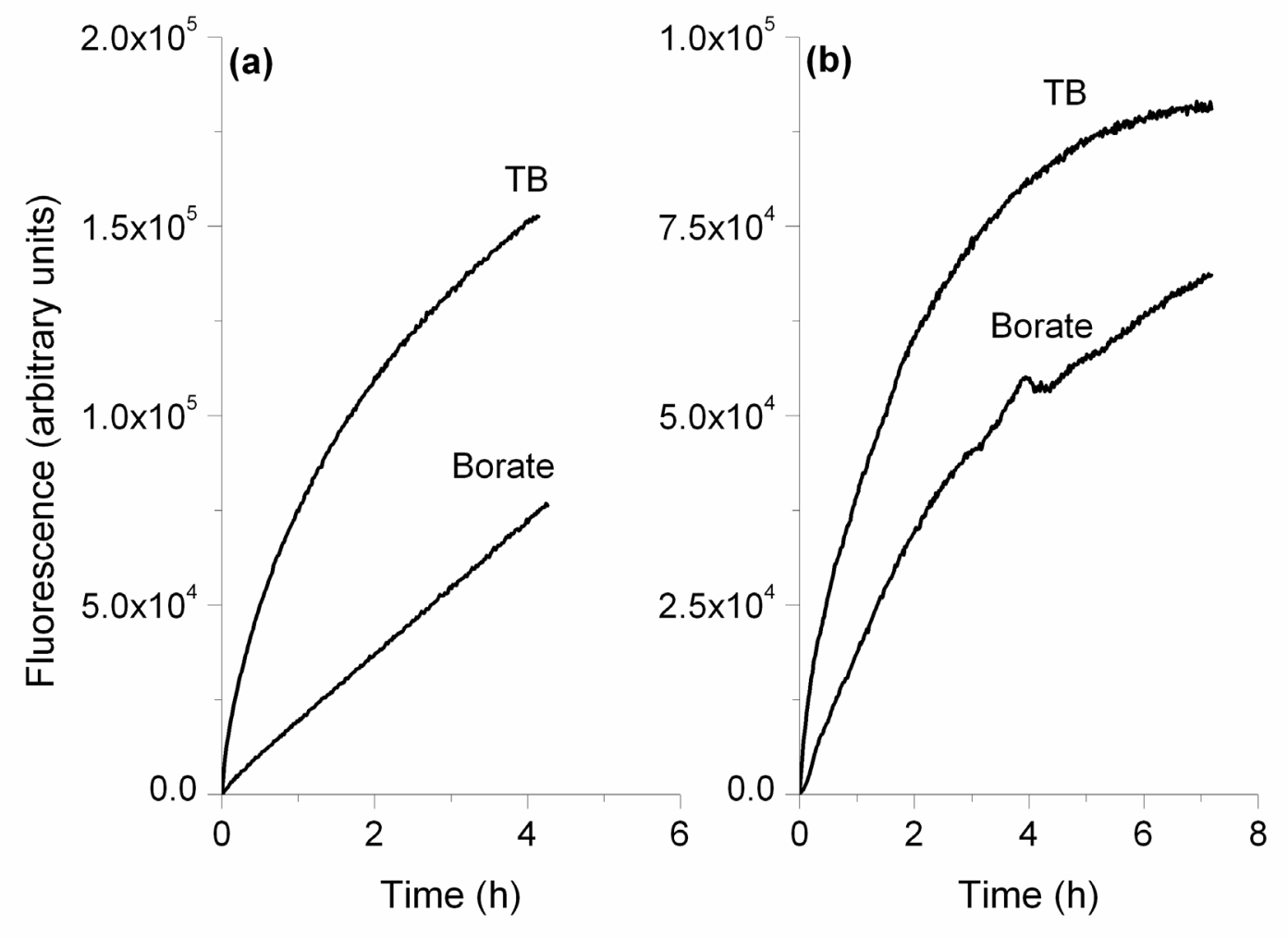

Figure S1. Sample kinetic curves for: a) adsorption of a mixed base oligonucleotide (6) on a MAA-modified QD in TB $(\mathrm{pH}=7.4)$ and borate buffer $(\mathrm{pH}=9.5)$; b) hybridization of a mixed base target oligonucleotide (5) with QD-1×DNA (1) conjugate in TB and borate buffer. In each case, note the faster kinetics in TB buffer.

\section{References}

1. Major, A.; Barzda, V.; Piunno, P.A.E., Musikhin, S.; Krull U.J. Opt. Exp. 2006, 14, 5285.

2. Azab, H.A.; Anwar, Z.M.; Sokar, M. J. Chem. Eng. Data. 2004, 49, 256.

3. Travers, A.A. Annu. Rev. Biochem. 1989, 58, 427-452.

4. Lin, C.H.; Sun, D., Hurley, L.H. Chem. Res. Toxicol. 1990, 4, 21. 